Internat. J. Math. \& Math. Sci.

Vol. 23, No. 11 (2000) 789-794

S0161171200003112

(C) Hindawi Publishing Corp.

\title{
COMPLETE CONVERGENCE FOR SUMS OF ARRAYS OF RANDOM ELEMENTS
}

\author{
SOO HAK SUNG
}

(Received 13 October 1997)

\begin{abstract}
Let $\left\{X_{n i}\right\}$ be an array of rowwise independent $B$-valued random elements and $\left\{a_{n}\right\}$ constants such that $0<a_{n} \uparrow \infty$. Under some moment conditions for the array, it is shown that $\sum_{i=1}^{n} X_{n i} / a_{n}$ converges to 0 completely if and only if $\sum_{i=1}^{n} X_{n i} / a_{n}$ converges to 0 in probability.
\end{abstract}

Keywords and phrases. Complete convergence, random elements, Banach space, rowwise independent.

2000 Mathematics Subject Classification. Primary 60B12, 60F15.

1. Introduction. Let $(B,\|\|)$ be a real separable Banach space. A separable Banach space $B$ is said to be of type $r, 1 \leq r \leq 2$, if there exists a constants $C_{r}$ such that

$$
E\left\|\sum_{i=1}^{n} X_{i}\right\|^{r} \leq C_{r} \sum_{i=1}^{n} E\left\|X_{i}\right\|^{r}
$$

for all independent $B$-valued random elements $X_{1}, \ldots, X_{n}$ with mean zero and finite $r$ th moments.

A sequence $\left\{X_{n}, n \geq 1\right\}$ of $B$-valued random elements is said to converge completely to zero if for each $\epsilon>0$,

$$
\sum_{n=1}^{\infty} P\left(\left\|X_{n}\right\|>\epsilon\right)<\infty .
$$

Note that complete convergence implies almost surely by the Borel-Cantelli lemma.

Now let $\left\{X_{n}, n \geq 1\right\}$ be a sequence of independent random variables. Let $\psi(t)$ be a positive, even and continuous function such that

$$
\frac{\psi(|t|)}{|t|} \uparrow \text { and } \frac{\psi(|t|)}{|t|^{2}} \downarrow \text { as }|t| \uparrow .
$$

Chung [3] strong law of large numbers (SLLN) states that if

$$
E X_{n}=0 \quad \text { for } n \geq 1, \quad \sum_{n=1}^{\infty} \frac{E \psi\left(\left|X_{n}\right|\right)}{\psi(n)}<\infty
$$

then

$$
\frac{\sum_{i=1}^{n} X_{i}}{n} \longrightarrow 0 \text { almost surely. }
$$


Recently, $\mathrm{Hu}$ and Taylor [6] proved Chung type SLLN for arrays of rowwise independent random variables. More specifically, let $\left\{X_{n i}, 1 \leq i \leq n, n \geq 1\right\}$ be an array of rowwise independent random variables and let $\left\{a_{n}, n \geq 1\right\}$ be a sequence of real numbers with $0<a_{n} \uparrow \infty$. Let $\psi(t)$ be a positive, even and continuous function such that

$$
\frac{\psi(|t|)}{|t|^{p}} \uparrow \text { and } \frac{\psi(|t|)}{|t|^{p+1}} \downarrow \text { as }|t| \uparrow
$$

for some integer $p \geq 2$. Furthermore, assume that

$$
\begin{gathered}
E X_{n i}=0 \quad \text { for } 1 \leq i \leq n, n \geq 1, \\
\sum_{n=1}^{\infty} \sum_{i=1}^{n} \frac{E \psi\left(\left|X_{n i}\right|\right)}{\psi\left(a_{n}\right)}<\infty, \\
\sum_{n=1}^{\infty}\left(\sum_{i=1}^{n} \frac{E X_{n i}^{2}}{a_{n}^{2}}\right)^{2 k}<\infty,
\end{gathered}
$$

where $k$ is a positive integer. Then the conditions (1.6), (1.7), (1.8), and (1.9) imply

$$
\frac{1}{a_{n}} \sum_{i=1}^{n} X_{n i} \longrightarrow 0 \text { almost surely. }
$$

Many classical theorems hold for $B$-valued random elements under the assumption that the weak law of large numbers (WLLN) holds (see, Kuelbs and Zinn [8], de Acosta [4], Choi and Sung [1, 2], Wang, Rao and Yang [10], Kuczmaszewska and Szynal [7], and Sung [9]).

In this paper, we apply de Acosta [4] inequality to obtain Hu and Taylor's [6] result in a general Banach space under the assumption that WLLN holds.

2. Main Result. To prove our main theorem, we need the following lemma which is due to de Acosta [4].

LEMMA 2.1. For each $p \geq 1$, there exists a positive constant $C_{p}$ such that for separable Banach space $B$ and any finite sequence $\left\{X_{i}, 1 \leq i \leq n\right\}$ of independent $B$-valued random elements with $E\left\|X_{i}\right\|^{p}<\infty(1 \leq i \leq n)$, the following inequalities hold.

(i) For $1 \leq p \leq 2$,

$$
E\left\|\left|\left\|\sum_{i=1}^{n} X_{i}\right\|-E\left\|\sum_{i=1}^{n} X_{i}\right\|\right|^{p} \leq C_{p} \sum_{i=1}^{n} E\right\| X_{i} \|^{p} .
$$

(ii) $\operatorname{For} p>2$,

$$
E\left|\left\|\sum_{i=1}^{n} X_{i}\right\|-E\left\|\sum_{i=1}^{n} X_{i}\right\|\right|^{p} \leq C_{p}\left[\left(\sum_{i=1}^{n} E\left\|X_{i}\right\|^{2}\right)^{p / 2}+\sum_{i=1}^{n} E\left\|X_{i}\right\|^{p}\right] .
$$

Throughout this paper, let $\psi(t)$ be a positive, even function such that

$$
\frac{\psi(|t|)}{|t|} \uparrow \text { and } \frac{\psi(|t|)}{|t|^{p}} \downarrow \text { as }|t| \uparrow
$$

for some $p \geq 1$. 
THEOREM 2.2. Let $\left\{X_{n i}, 1 \leq i \leq n, n \geq 1\right\}$ be an array of rowwise independent $B$-valued random elements and $\left\{a_{n}, n \geq 1\right\}$ constants such that $0<a_{n} \uparrow \infty$. Assume that

$$
\begin{aligned}
& \sum_{n=1}^{\infty} \sum_{i=1}^{n} \frac{E \psi\left(\left\|X_{n i}\right\|\right)}{\psi\left(a_{n}\right)}<\infty, \\
& \sum_{n=1}^{\infty}\left(\sum_{i=1}^{n} \frac{E\left\|X_{n i}\right\|^{2}}{a_{n}^{2}}\right)^{s}<\infty
\end{aligned}
$$

for some $s>0$. Then the following statements are equivalent.

(i) $\left(1 / a_{n}\right) \sum_{i=1}^{n} X_{n i} \rightarrow 0$ in $L^{1}$.

(ii) $\left(1 / a_{n}\right) \sum_{i=1}^{n} X_{n i} \rightarrow 0$ completely.

(iii) $\left(1 / a_{n}\right) \sum_{i=1}^{n} X_{n i} \rightarrow 0$ almost surely.

(iv) $\left(1 / a_{n}\right) \sum_{i=1}^{n} X_{n i} \rightarrow 0$ in probability.

Proof. (i) $\Longrightarrow$ (ii). Define $Y_{n i}=X_{n i} I\left(\left\|X_{n i}\right\| \leq a_{n}\right)$ and $Z_{n i}=X_{n i} I\left(\left\|X_{n i}\right\|>a_{n}\right)$. Since $\psi(|t|) /|t|$ is an increasing function of $|t|$, we have by (2.4) that

$$
\begin{aligned}
\sum_{n=1}^{\infty} \frac{1}{a_{n}} E\left\|\sum_{i=1}^{n} Z_{n i}\right\| & \leq \sum_{n=1}^{\infty} \frac{1}{a_{n}} \sum_{i=1}^{n} E\left\|Z_{n i}\right\| \\
& \leq \sum_{n=1}^{\infty} \frac{1}{\psi\left(a_{n}\right)} \sum_{i=1}^{n} E \psi\left(\left\|Z_{n i}\right\|\right) \leq \sum_{n=1}^{\infty} \sum_{i=1}^{n} \frac{E \psi\left(\left\|X_{n i}\right\|\right)}{\psi\left(a_{n}\right)}<\infty .
\end{aligned}
$$

It follows that

$$
\frac{1}{a_{n}} \sum_{i=1}^{n} Z_{n i} \longrightarrow 0 \text { completely. }
$$

The proof will be completed by showing that

$$
\frac{1}{a_{n}} \sum_{i=1}^{n} Y_{n i} \longrightarrow 0 \text { completely. }
$$

From (i) and (2.6), we have

$$
\begin{aligned}
\frac{1}{a_{n}} E\left\|\sum_{i=1}^{n} Y_{n i}\right\| & =\frac{1}{a_{n}} E\left\|\sum_{i=1}^{n}\left(X_{n i}-Z_{n i}\right)\right\| \\
& \leq \frac{1}{a_{n}} E\left\|\sum_{i=1}^{n} X_{n i}\right\|+\frac{1}{a_{n}} E\left\|\sum_{i=1}^{n} Z_{n i}\right\| \rightarrow 0 .
\end{aligned}
$$

Thus, to prove (2.8), it is enough to show that

$$
\frac{1}{a_{n}}\left\|\sum_{i=1}^{n} Y_{n i}\right\|-\frac{1}{a_{n}} E\left\|\sum_{i=1}^{n} Y_{n i}\right\| \longrightarrow 0 \text { completely. }
$$

First consider the case of $1 \leq p \leq 2$. From Markov's inequality and Lemma 2.1(i), we have 


$$
\begin{aligned}
\sum_{n=1}^{\infty} P\left(\mid \frac{1}{a_{n}} \|\right. & \left.\sum_{i=1}^{n} Y_{n i}\left\|-\frac{1}{a_{n}} E\right\| \sum_{i=1}^{n} Y_{n i} \| \mid>\epsilon\right) \\
& \leq \frac{1}{\epsilon^{p}} \sum_{n=1}^{\infty} \frac{1}{a_{n}^{p}} E\left\|\left|\sum_{i=1}^{n} Y_{n i}\|-E\| \sum_{i=1}^{n} Y_{n i} \|\right|^{p}\right. \\
& \leq \frac{C_{p}}{\epsilon^{p}} \sum_{n=1}^{\infty} \sum_{i=1}^{n} \frac{E\left\|Y_{n i}\right\|^{p}}{a_{n}^{p}} \leq \frac{C_{p}}{\epsilon^{p}} \sum_{n=1}^{\infty} \sum_{i=1}^{n} \frac{E \psi\left(\left\|Y_{n i}\right\|\right)}{\psi\left(a_{n}\right)} \\
& \leq \frac{C_{p}}{\epsilon^{p}} \sum_{n=1}^{\infty} \sum_{i=1}^{n} \frac{E \psi\left(\left\|X_{n i}\right\|\right)}{\psi\left(a_{n}\right)}<\infty
\end{aligned}
$$

since $\psi(|t|) /|t|^{p} \downarrow$ and (2.4). Thus (2.10) holds.

Now consider the case of $p>2$. Note that $\psi(|t|) /|t|^{p} \downarrow$ implies $\psi(|t|) /|t|^{q} \downarrow$ for each $q \geq p$. Let $q=\max \{p, 2 s\}$. Then we have by Markov's inequality and Lemma 2.1 (ii) that

$$
\begin{aligned}
\sum_{n=1}^{\infty} P\left(\mid \frac{1}{a_{n}}\right. & \left.\left\|\sum_{i=1}^{n} Y_{n i}\right\|-\frac{1}{a_{n}} E\left\|\sum_{i=1}^{n} Y_{n i}\right\| \mid>\epsilon\right) \\
& \leq \frac{1}{\epsilon^{q}} \sum_{n=1}^{\infty} \frac{1}{a_{n}^{q}} E\left|\left\|\left|\sum_{i=1}^{n} Y_{n i}\|-E\| \sum_{i=1}^{n} Y_{n i} \|\right|^{q}\right.\right. \\
& \leq \frac{C_{q}}{\epsilon^{q}} \sum_{n=1}^{\infty} \frac{1}{a_{n}^{q}}\left[\left(\sum_{i=1}^{n} E\left\|Y_{n i}\right\|^{2}\right)^{q / 2}+\sum_{i=1}^{n} E\left\|Y_{n i}\right\|^{q}\right] \\
& =\frac{C_{q}}{\epsilon^{q}} \sum_{n=1}^{\infty}\left(\frac{\sum_{i=1}^{n} E\left\|Y_{n i}\right\|^{2}}{a_{n}^{2}}\right)^{q / 2}+\frac{C_{q}}{\epsilon^{q}} \sum_{n=1}^{\infty} \frac{1}{a_{n}^{q}} \sum_{i=1}^{n} E\left\|Y_{n i}\right\|^{q} .
\end{aligned}
$$

Since $q \geq p, \psi(|t|) /|t|^{p} \downarrow$ implies $\psi(|t|) /|t|^{q} \downarrow$, and so

$$
\sum_{n=1}^{\infty} \frac{1}{a_{n}^{q}} \sum_{i=1}^{n} E\left\|Y_{n i}\right\|^{q} \leq \sum_{n=1}^{\infty} \sum_{i=1}^{n} \frac{E \psi\left(\left\|Y_{n i}\right\|\right)}{\psi\left(a_{n}\right)} \leq \sum_{n=1}^{\infty} \sum_{i=1}^{n} \frac{E \psi\left(\left\|X_{n i}\right\|\right)}{\psi\left(a_{n}\right)}<\infty .
$$

Also,

$$
\begin{aligned}
\sum_{n=1}^{\infty}\left(\frac{\sum_{i=1}^{n} E\left\|Y_{n i}\right\|^{2}}{a_{n}^{2}}\right)^{q / 2} & \leq\left[\sum_{n=1}^{\infty}\left(\frac{\sum_{i=1}^{n} E\left\|Y_{n i}\right\|^{2}}{a_{n}^{2}}\right)^{s}\right]^{q / 2 s} \\
& \leq\left[\sum_{n=1}^{\infty}\left(\frac{\sum_{i=1}^{n} E\left\|X_{n i}\right\|^{2}}{a_{n}^{2}}\right)^{s}\right]^{q / 2 s}<\infty,
\end{aligned}
$$

since $q \geq 2 s$ and (2.5). Combining (2.12), (2.13), and (2.14) yields (2.10). Thus (i) $\Longrightarrow$ (ii) is proved. Since the implications (ii) $\Longrightarrow$ (iii) and (iii) $\Longrightarrow$ (iv) are obvious, it remains to show that (iv) $\Longrightarrow$ (i).

Assume that (iv) holds. From Lemma 2.1(i) and (2.5)

$$
E\left|\frac{1}{a_{n}}\left\|\sum_{i=1}^{n} X_{n i}\right\|-\frac{1}{a_{n}} E\left\|\sum_{i=1}^{n} X_{n i}\right\|\right|^{2} \leq \frac{C_{2}}{a_{n}^{2}} \sum_{i=1}^{n} E\left\|X_{n i}\right\|^{2} \longrightarrow 0,
$$

which entails

$$
\frac{1}{a_{n}}\left\|\sum_{i=1}^{n} X_{n i}\right\|-\frac{1}{a_{n}} E\left\|\sum_{i=1}^{n} X_{n i}\right\| \longrightarrow 0 \text { in probability. }
$$


It follows by (iv) that $E\left\|\sum_{i=1}^{n} X_{n i}\right\| / a_{n} \longrightarrow 0$, and so (i) holds. Thus the proof of Theorem 2.2 is completed.

The following theorem states that Theorem 2.2 holds even if the condition (2.5) is replaced by

$$
\sum_{n=1}^{\infty}\left(\sum_{i=1}^{n} \frac{E\left\|X_{n i}\right\|^{r}}{a_{n}^{r}}\right)^{s}<\infty
$$

for some $1 \leq r \leq 2$ and $s>0$.

THEOREM 2.3. Let $\left\{X_{n i}, 1 \leq i \leq n, n \geq 1\right\}$ be an array of rowwise independent $B$-valued random elements and $\left\{a_{n}, n \geq 1\right\}$ constants such that $0<a_{n} \uparrow \infty$. Assume that (2.4) and (2.17) hold. Then the following statements are equivalent.

(i) $\left(1 / a_{n}\right) \sum_{i=1}^{n} X_{n i} \rightarrow 0$ in $L^{1}$.

(ii) $\left(1 / a_{n}\right) \sum_{i=1}^{n} X_{n i} \rightarrow 0$ completely.

(iii) $\left(1 / a_{n}\right) \sum_{i=1}^{n} X_{n i} \rightarrow 0$ almost surely.

(iv) $\left(1 / a_{n}\right) \sum_{i=1}^{n} X_{n i} \rightarrow 0$ in probability.

Proof. Let $\left\{Y_{n i}\right\}$ and $\left\{Z_{n i}\right\}$ be as in the proof of Theorem 2.2. From the proof of (i) $\Longrightarrow$ (ii) in Theorem 2.2, we have

$$
\sum_{n=1}^{\infty} \frac{1}{a_{n}} E\left\|\sum_{i=1}^{n} Z_{n i}\right\|<\infty
$$

which implies $\sum_{i=1}^{n} Z_{n i} / a_{n} \rightarrow 0$ in $L^{1}$, completely, almost surely, and in probability. Hence, it is enough to show that

$$
\begin{aligned}
\frac{1}{a_{n}} \sum_{i=1}^{n} Y_{n i} \longrightarrow 0 \text { in } L^{1} & \Longleftrightarrow \frac{1}{a_{n}} \sum_{i=1}^{n} Y_{n i} \longrightarrow 0 \text { completely } \\
& \Leftrightarrow \frac{1}{a_{n}} \sum_{i=1}^{n} Y_{n i} \longrightarrow 0 \text { almost surely } \\
& \Leftrightarrow \frac{1}{a_{n}} \sum_{i=1}^{n} Y_{n i} \longrightarrow 0 \text { in probability. }
\end{aligned}
$$

Since $Y_{n i}=X_{n i} I\left(\left\|X_{n i}\right\| \leq a_{n}\right)$, it follows that $E \psi\left(\left\|Y_{n i}\right\|\right) \leq E \psi\left(\left\|X_{n i}\right\|\right)$ and

$$
\sum_{n=1}^{\infty}\left(\sum_{i=1}^{n} \frac{E\left\|Y_{n i}\right\|^{2}}{a_{n}^{2}}\right)^{s} \leq \sum_{n=1}^{\infty}\left(\sum_{i=1}^{n} \frac{E\left\|Y_{n i}\right\|^{r}}{a_{n}^{r}}\right)^{s} \leq \sum_{n=1}^{\infty}\left(\sum_{i=1}^{n} \frac{E\left\|X_{n i}\right\|^{r}}{a_{n}^{r}}\right)^{s} .
$$

Thus $\left\{Y_{n i}\right\}$ satisfies the conditions of Theorem 2.2, and so (2.19) holds by Theorem 2.2.

COROLLARY 2.4. Let $\left\{X_{n i}, 1 \leq i \leq n, n \geq 1\right\}$ be an array of rowwise independent $B$-valued random elements and $\left\{a_{n}, n \geq 1\right\}$ constants such that $0<a_{n} \uparrow \infty$. Assume that $E X_{n i}=0$ and $B$ is of type $r(1 \leq r \leq 2)$. Then (2.4) and (2.17) imply that

$$
\frac{1}{a_{n}} \sum_{i=1}^{n} X_{n i} \rightarrow 0 \text { almost surely. }
$$


Proof. By Theorem 2.3, it is enough to show that

$$
\frac{1}{a_{n}} \sum_{i=1}^{n} X_{n i} \longrightarrow 0 \text { in } L^{1} .
$$

Since $B$ is of type $r$ and $E X_{n i}=0$, it follows by (2.17) that

$$
E\left\|\frac{1}{a_{n}} \sum_{i=1}^{n} X_{n i}\right\|^{r} \leq \frac{C_{r}}{a_{n}^{r}} \sum_{i=1}^{n} E\left\|X_{n i}\right\|^{r} \longrightarrow 0,
$$

and so (2.22) holds.

REMARK 2.5. The condition (2.3) is weaker than (1.6). Hu and Chung [5] proved Corollary 2.4 under the stronger condition (1.6).

\section{REFERENCES}

[1] B. D. Choi and S. H. Sung, On Chung's strong law of large numbers in general Banach spaces, Bull. Austral. Math. Soc. 37 (1988), no. 1, 93-100. MR 89b:60018. Zbl 628.60012.

[2] __ On Teicher's strong law of large numbers in general Banach spaces, Probab. Math. Statist. 10 (1989), no. 1, 137-142. MR 90f:60013. Zbl 686.60007.

[3] K. L. Chung, Note on some strong laws of large numbers, Amer. J. Math. 69 (1947), 189192. MR 8,471a. Zbl 034.07103.

[4] A. de Acosta, Inequalities for B-valued random vectors with applications to the strong law of large numbers, Ann. Probab. 9 (1981), no. 1, 157-161. MR 83c:60009. Zbl 449.60002.

[5] T. C. Hu and H. C. Chang, Strong laws of large numbers for arrays of random elements, Soochow J. Math. 20 (1994), no. 4, 587-594. MR 95k:60012. Zbl 861.60014.

[6] T. C. Hu and R. L. Taylor, On the strong law for arrays and for the bootstrap mean and variance, Internat. J. Math. Math. Sci. 20 (1997), no. 2, 375-382. MR 97k:60011. Zbl 883.60024.

[7] A. Kuczmaszewska and D. Szynal, On complete convergence in a Banach space, Internat. J. Math. Math. Sci. 17 (1994), no. 1, 1-14. MR 95d:60012. Zbl 798.60006.

[8] J. Kuelbs and J. Zinn, Some stability results for vector valued random variables, Ann. Probab. 7 (1979), no. 1, 75-84. MR 80h:60014. Zbl 399.60007.

[9] S. H. Sung, Complete convergence for weighted sums of arrays of rowwise independent $B$-valued random variables, Stochastic Anal. Appl. 15 (1997), no. 2, 255-267. MR 98c:60007. Zbl 902.60011.

[10] X. C. Wang, M. B. Rao, and X. Y. Yang, Convergence rates on strong laws of large numbers for arrays of rowwise independent elements, Stochastic Anal. Appl. 11 (1993), no. 1, 115-132. MR 94a:60007. Zbl 764.60037.

Sung: Department of Applied Mathematics, Pai Chai University, Taejon 302-735, SOUTH KOREA

E-mail address: sungsh@mai 1 . paichai .ac. kr 


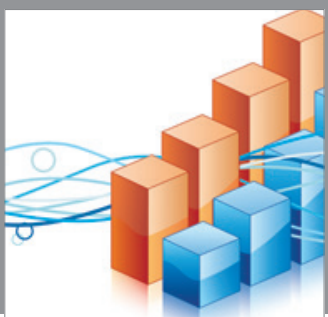

Advances in

Operations Research

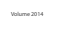

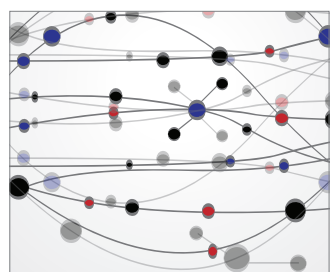

\section{The Scientific} World Journal
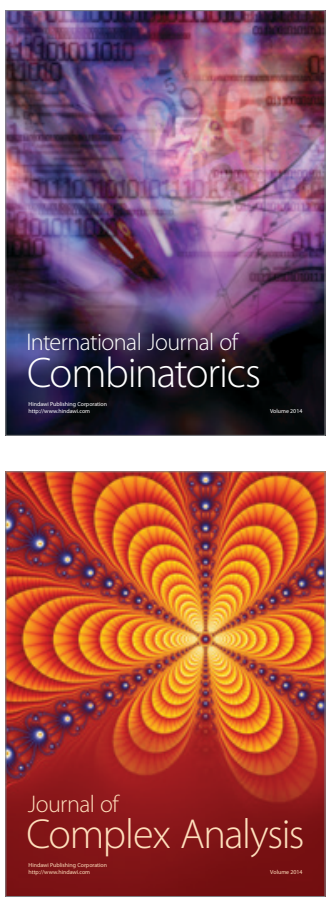

International Journal of

Mathematics and

Mathematical

Sciences
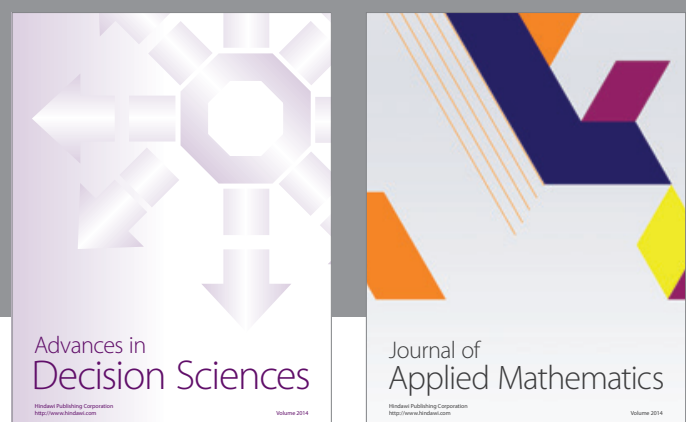

Journal of

Applied Mathematics
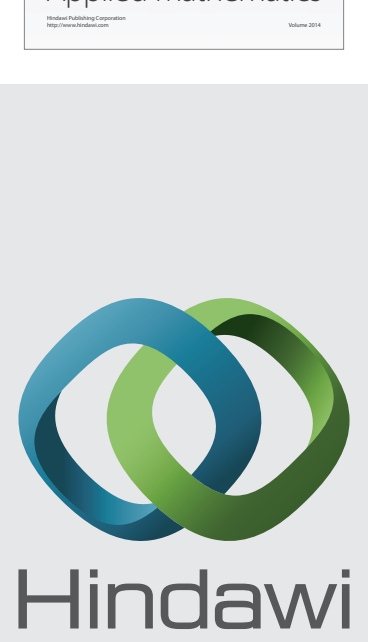

Submit your manuscripts at http://www.hindawi.com
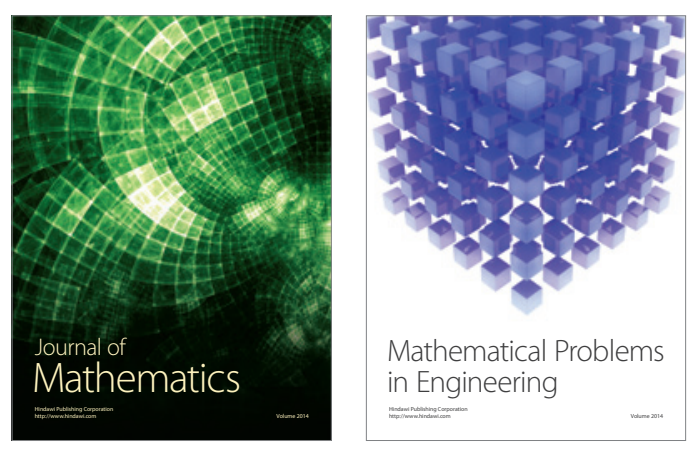

Mathematical Problems in Engineering
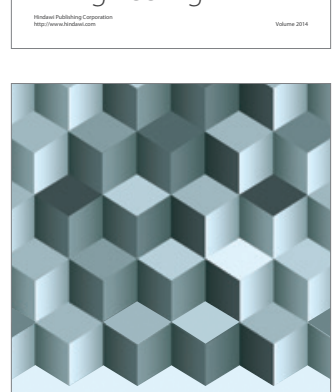

Journal of

Function Spaces
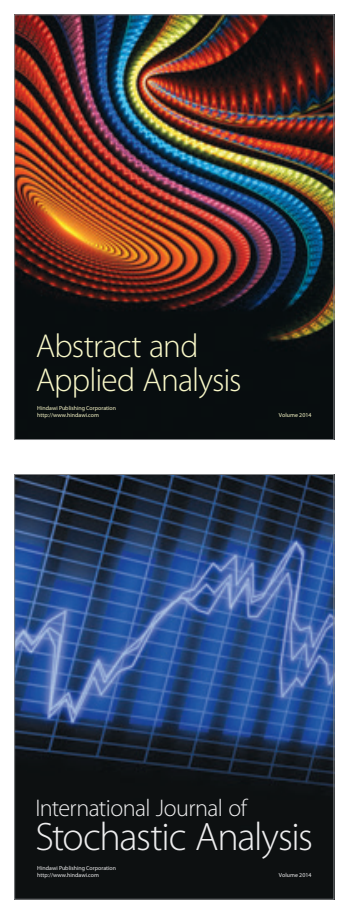

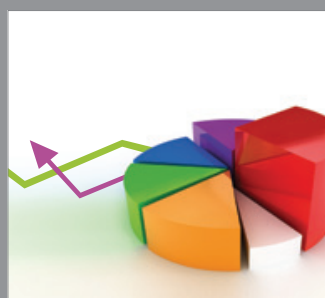

ournal of

Probability and Statistics

Promensencen
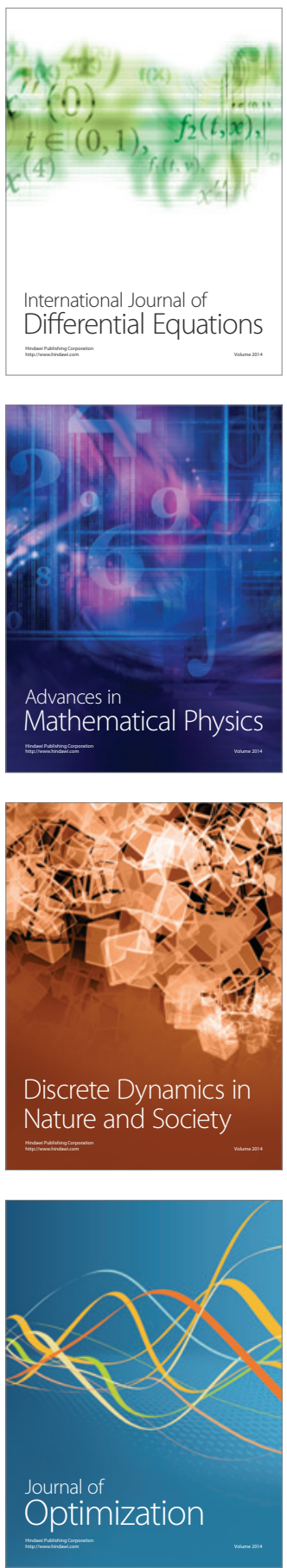